\title{
CONDENSED JULIA SETS, WITH AN APPLICATION TO A FRACTAL LATTICE MODEL HAMILTONIAN
}

\author{
BY
}

\author{
M. F. BARNSLEY, J. S. GERONIMO ${ }^{1}$ AND A. N. HARRINGTON ${ }^{1}$
}

\begin{abstract}
The Julia set for the complex rational map $z \rightarrow z^{2}-\lambda+\varepsilon / z$, where $\lambda$ and $\varepsilon$ are complex parameters, is considered in the limit as $\varepsilon \rightarrow 0$. The result is called the condensed Julia set for $z \rightarrow\left(z^{3}-\lambda z\right) / z$. The limit of balanced measures, associated functional equations and orthogonal polynomials are considered; it is shown, for example, that for $\lambda \geqslant 2$ the moments, orthogonal polynomials, and associated Jacobi matrix $\mathscr{J}$ can be calculated explicitly and are not those belonging to $z^{2}-\lambda$. The spectrum of $\mathscr{J}$ consists of a point spectrum $P$ together with its derived set. The latter is the Julia set for $z^{2}-\lambda$, and carries none of the spectral mass when $\lambda>2$. When $\lambda=2, P$ is dense in $[-2,2]$. A similar condensation in the case $\lambda=15 / 4$ leads to a system which corresponds precisely to the spectrum and density of states of a two-dimensional Sierpinski gasket model Schrödinger equation.

The basic ideas about condensation of Julia sets in general are described. If $R(z)$ is a rational transformation of degree greater than one, then condensation can be attached to
\end{abstract}

$$
z \rightarrow R(z)+\varepsilon \sum_{i=1}^{k}\left(z-a_{i}\right)^{-\gamma_{i}},
$$

where the $\gamma_{i}$ 's and $k$ are finite positive integers and the $a_{i}$ 's are complex numbers. If $\infty$ is an indifferent or attractive fixed point of $R(z)$, then all of the moments of the associated condensed balanced measure can be calculated explicitly, as can the orthogonal polynomials when the condensed Julia set is real. Sufficient conditions for the condensed measure $\sigma$ to be a weak limit of the balanced measures $\mu_{\varepsilon}$ are given. Functional equations connected to the condensed measure are derived, and it is noted that their form typifies those encountered in statistical physics, in connection with partition functions for Ising hierarchical models.

1. Introduction. Among the many rich directions of study, connected more or less with Julia sets (see, for example, [DK, Do, DoH, F, Gu, H, J. M1, M2, MSS, Re, Ru, S1-4, T]), there is the theory of moments of balanced measures on these sets [AR, BaBM, BGH1-7, BH, BGM, BMM1, BMM2, BM, Br, D, FLM, Ma, PK]. The balanced measure attaches equal weight to each of the inverse branches of the rational transformation which generates the Julia set. This implies that its power moments can be calculated explicitly, not only in the case where the generating rational transformation is a polynomial [BGH1, BMM2], but also under broadly satisfied additional hypotheses, in the case of rational transformations [BH, BGM].

Received by the editors November 28, 1983 and, in revised form, February 1, 1984.

1980 Mathematics Subject Classification. Primary 30D05, 82A43; Secondary 30E05, 39B10.

${ }^{1}$ Supported in part by NSF grant number MCS 8203325 . 
The explicit computability of moments has led to applications in a number of areas including Padé approximation, special functions and orthogonal polynomials. Also of particular interest has been the discovery of relationships between this topic and model Schrödinger equations on one-dimensional lattices, involving almost periodic couplings. Various such models are now understood in a very detailed way: their spectra are Julia sets, with spectral densities given by the associated balanced measures; and the analysis can be made complete because the geometry and topology of the Julia sets involved are well understood, as is the dynamics of these sets under the generating rational transformation, and the relationship between the generating rational transformation and the orthogonal polynomials which themselves fix the behavior of the (nonlocalized) eigenfunctions. Thus, the exact computability of moments has already made a good addition to theory of almost periodic operators [BT, AS].

Also, the functional equations obeyed by the generating functions of the moments of balanced measures, and certain transforms of these equations, have been shown to be of interest in statistical physics, in connection with Ising-like hierarchical models [DDI, NF, Dh, BO, KG].

One apparent weakness, for applications, in the linear operators associated with balanced measures is that their spectra are either absolutely or singular continuous and thus no models are obtained which involve point spectra and bound state eigenfunctions. It was this shortcoming which, in part, motivated the research described in this paper.

Here we report a new class of sets and measures, which we call condensed Julia sets and condensed measures, for which a number of the important properties of Julia sets and balanced measures are preserved. A condensed Julia set $C$ and condensed measure $\sigma$ are obtained formally by taking limits as $\varepsilon \rightarrow 0$ of the family of Julia sets $J_{\varepsilon}$ and balanced measures $\mu_{\varepsilon}$ attached to a family of rational transformations of the form

$$
R_{\varepsilon}(z)=R_{1}(z)+\varepsilon R_{2}(z),
$$

where $R_{1}(z)$ and $R_{2}(z)$ are rational transformations.

Under appropriate conditions, we analyze the structures of $C$ and $\sigma$, and show how the moments of $\sigma$ may be calculated explicitly in terms of the coefficients in $R_{1}(z)$ and $R_{2}(z)$. The functional equation obeyed by the moment generating function for $\sigma$ can then be displayed. When $C \subset \mathbf{R}$, the orthogonal polynomials for $\sigma$ and the recurrence formulas among them can be given. The associated Jacobi matrix, with spectrum $C$ and spectral density $\sigma$, now possesses eigenvalues. This considerably broadens the areas within which applications may be found: it has led in particular to an application in connection with a model Schrödinger equation on a Sierpinski gasket.

In $\$ 2$ we review the theory of balanced measures on Julia sets, and show how the moments of such measures $\mu$ may be calculated. The formulation of a selfadjoint operator $\mathscr{J}$, a Jacobi matrix, whose spectral density is $\mu$, is briefly described. The functional equation obeyed by the spectral function, the moment generating function and the associated potential function are derived. 
We begin $\$ 3$ with an example of the condensation process, which involves the Julia sets $J_{\varepsilon}$ and balanced measures $\mu_{\varepsilon}$ for the family of rational transformations

$$
R_{\varepsilon}(z)=z^{2}-\lambda+\varepsilon / z, \quad \lambda>2,
$$

as $\varepsilon \rightarrow 0$. The resulting condensed Julia set $C$, condensed measure $\sigma$ and associated structures are contrasted with the Julia set $J_{0}$, balanced measure $\mu_{0}$ and associated structures corresponding to $R_{0}(z)$. In particular, we compare the nature of the Jacobi matrix for $\sigma$ with that for $\mu_{0}$, which is of interest because the latter is known to be limit-periodic and to obey a renormalization equation [BBM].

We conclude $\$ 3$ by analyzing the general situation: a condensed Julia set and condensed measure $\sigma$ can be associated with the unreduced rational function

$$
R(z) \prod_{i=1}^{k}\left(z-a_{i}\right)^{\gamma_{i}} / \prod_{i=1}^{k}\left(z-a_{i}\right)^{\gamma_{i}},
$$

where the $\gamma_{i}$ 's and $n$ are finite positive integers, the $a_{i}$ 's are complex numbers and $R(z)$ is a rational transformation of degree greater than one. If the Julia set $J$ for $R(z)$ is hyperbolic [BI], $\gamma_{i}=1$ for $i=1, \ldots, N$, and if the points $a_{i}$ belong neither to $J$ nor to any $k$-cycle of $R(z)$, then the condensation may be viewed as coming from

$$
R_{\varepsilon}(z)=R(z)+\varepsilon \sum_{i=1}^{k}\left(z-a_{i}\right)^{\gamma_{i}}
$$

in the sense of weak limits. In any case $\sigma$ is well defined and, if $\infty$ is an attractive or indifferent fixed point of $R(z)$, all of its moments exist and can be calculated recursively.

In $\$ 4$ we recall the details associated with a model Schrödinger equation on a Sierpinski gasket, which was analyzed recently by Kadanoff et al. [DABK]. For the considered model we show that the spectrum of the Schrödinger operator is essentially the condensed Julia set for

$$
z \rightarrow-z \cdot(z+3)+\varepsilon\left(z^{2}-1\right)^{-1}
$$

whilst the density of states is a linear combination of the condensed measures for

$$
\begin{aligned}
& z \rightarrow-z \cdot(z+3)+\varepsilon(z-1), \\
& z \rightarrow-z \cdot(z+3)+\varepsilon(z+1),
\end{aligned}
$$

together with a contribution at $z=2$. The functional equation obeyed by the associated spectral function is thus deduced, and shown to be the one obtained by Rammal [R1, R2] for a similar model.

In conclusion we suspect that many models with an exact renormalization group are associated with condensed Julia sets for appropriate rational transformations. In view of the limiting connection between condensed and balanced measures, and the fact that the latter are associated in some proven cases with almost periodicity, we believe that similar connections between renormalizable lattice models and almost periodicity, or at least ideas in the same bailiwick, should appear. Conversely, information about renormalizable lattice models and their eigenfunctions should give information about orthogonal polynomials associated with rational transforma. tions, at least in the condensation limit. 
2. Balanced measures on Julia sets. We recall the theory of balanced measures on Julia sets, and we derive the associated functional equations. What is given here is essential to understanding the subsequent parts of the paper.

Let $R(z): \hat{\mathbf{C}} \rightarrow \hat{\mathbf{C}}$ denote a rational transformation of the extended complex plane $\hat{\mathbf{C}}=\mathbf{C} \cup\{\infty\}$ into itself, of degree $N>1$. Then $R(z)=P(z) / Q(z)$ where $P$ and $Q$ are nontrivial polynomials, with no common factors, and $N=\max \{\operatorname{deg} P, \operatorname{deg} Q\}$. Let $J$ denote the Julia set for $R(z) . J$ is equal to the set of points at which the sequence of functions $\left\{R^{n}(z)\right\}_{n=0}^{\infty}$ is not normal, where $R^{0}(z)=z$ and $R^{n+1}(z)=$ $R^{n}(R(z))$ for $n=0,1,2, \ldots$ A family of analytic functions $F$ is said to be normal at $a \in \hat{\mathbf{C}}$ if there is a neighborhood of $a$ such that from any infinite sequence of functions of $F$, one can extract an infinite subsequence which converges uniformly.

Equivalently, the Julia set $J$ can be defined to be the closure of the set of all repulsive $k$-cycles of $R$ for all finite positive integers $k$. A $k$-cycle of $R$ is a set of $k$ distinct points $z_{i} \in \hat{\mathbf{C}}$ such that $z_{2}=R\left(z_{1}\right), z_{3}=R\left(z_{2}\right), \ldots, z_{k}=R\left(z_{k-1}\right)$, and $z_{1}=$ $R\left(z_{k}\right)$. The $k$-cycle is called repulsive when $\left|d R^{k}(z) / d z\right|_{z=z_{1}}>1$.

$J$ is a nondenumerable closed set which contains no isolated points. It either has no interior or else is $\hat{\mathbf{C}}$. It is completely invariant under $R$, namely $R^{-1}(J)=J$, where $R^{-1}$ denotes the inverse of $R$. We will write $\left\{R_{i}^{-1}(z)\right\}_{i=1}^{N}$ to denote a complete assignment of the branches of $R^{-1}(z)$.

Associated with the action of $R$ on $\hat{\mathbf{C}}$ there is a unique measure $\mu$, called the balanced measure for $R$, with the properties that $\mu(\hat{\mathbf{C}})=1$ and

$$
\mu(E)=N \mu\left(R_{i}^{-1} E\right) \text { for } i=1,2, \ldots, N,
$$

for all Borel subsets of $\hat{\mathbf{C}}$ [D]. The support of $\mu$ is $J$, and it associates no measure with any single point. A consequence of (1) is that if $f \in L_{1}(\hat{\mathbf{C}}, \mu)$, then

$$
\int_{\hat{\mathbf{C}}} f(z) d \mu(z)=\frac{1}{N} \int_{\hat{\mathbf{C}}} \sum_{i=1}^{N} f\left(R_{i}^{-1}(z)\right) d \mu(z) .
$$

A sequence of measures $\left\{\mu^{(n)}\right\}_{n=0}^{\infty}$ which converges weakly to $\mu$ can be constructed by starting from any probability measure $\mu^{(0)}$ on $\hat{\mathbf{C}}$ and defining inductively

$$
\mu^{(n+1)}\left(R_{i}^{-1} E\right)=(1 / N) \mu^{(n)}(E) \text { for } i=1,2, \ldots, N,
$$

for all Borel subsets $E$ of $\hat{\mathbf{C}}$.

Computation of the moments of $\mu$. In $[\mathbf{B H}]$ it is proved that if

$$
R(z)=\frac{z^{N}+a_{1} z^{N-1}+\cdots+a_{N}}{b_{1} z^{N-1}+b_{2} z^{N-2}+\cdots+b_{N}},
$$

where not all of the $b_{i}$ 's are zero, then $z^{n} \in L_{1}(\hat{\mathbf{C}}, \mu)$ for all $n=0,1,2, \ldots$ if and only if $\left|b_{1}\right| \leqslant 1$. This being the case, all of the moments $M_{n}=\int z^{n} d \mu(z), n=$ $0,1,2, \ldots$, can be calculated resursively as follows.

Define

$$
C_{k}(z)= \begin{cases}a_{k}-z b_{k} & \text { if } k=1,2, \ldots, N \\ 0 & \text { if } k=N+1, N+2, \ldots\end{cases}
$$


and

$$
S_{k}(z)=-k C_{k}(z)-\sum_{j=1}^{k-1} S_{k-j}(z) C_{k}(z) \text { for } k=1,2, \ldots
$$

If one now writes

$$
S_{m}(z)=\sum_{j=0}^{m} \Gamma_{m j} z^{j},
$$

where the $\Gamma_{m j}$ 's are independent of $z$, then

$$
M_{n}=\frac{1}{N-b_{1}^{m}} \sum_{j=0}^{m-1} \Gamma_{m j} M_{j} \text { for } m=1,2,3, \ldots
$$

This method for computing the moments $\left\{M_{n}\right\}_{1}^{\infty}$ is proved as follows. Using the property (2) of $\mu$ we have

$$
M_{m}=\int_{\hat{\mathbf{C}}} z^{m} d \mu(z)=\frac{1}{N} \int_{\hat{\mathbf{C}}} \sum_{j=1}^{N}\left(R_{j}^{-1}(z)\right)^{m} d \mu(z) .
$$

But $S_{m}(z)=\sum_{j=1}^{N}\left(R_{j}^{-1}(z)\right)^{m}$ is just the $m$ th symmetric function associated with the roots of the polynomial equation in $W$,

$$
W^{N}+C_{1}(z) W^{N-1}+\cdots+C_{N}(z)=0,
$$

and these functions can be calculated recursively using (4), [G]. $S_{m}(z)$ is found to be a polynomial in $z$ of degree at most $m$, and can be written in the form (5) wherein $\Gamma_{m m}=b_{1}^{m}$. Upon substituting into (7) we obtain

$$
N M_{m}=\int_{\hat{\mathbf{C}}} S_{m}(z) d \mu(z)=\sum_{j=0}^{m-1} \Gamma_{m j} M_{j}+b_{1}^{m} M_{m}
$$

from which (6) follows.

Orthogonal polynomials and Jacobi matrices. Let $R(z)$ be given by (*), and assume $J \subset \mathbf{R} \cup\{\infty\}$. Then from the moments $\left\{M_{n}\right\}_{n=0}^{\infty}$ one can calculate all of the orthogonal monic polynomials associated with $\mu$. These are $\left\{P_{m}(z)\right\}_{m=0}^{\infty}$, where $P_{m}(z)$ is a polynomial of degree $m$ and leading coefficient unity, such that

$$
\int_{J} P_{n}(z) P_{m}(z) d \mu(z)=0 \text { for } m \neq n .
$$

It is straightforward to verify $[\mathbf{S z}]$ that

$$
P_{n}(z)=\left|\begin{array}{ccc}
M_{0} & \ldots & M_{n} \\
\vdots & & \vdots \\
M_{n-1} & \cdots & M_{2 n-1} \\
1 & \cdots & z^{n}
\end{array}\right| \div\left|\begin{array}{ccc}
M_{0} & \ldots & M_{n} \\
\vdots & & \vdots \\
M_{n-1} & \cdots & M_{2 n-2}
\end{array}\right|
$$

Since the support of $\mu$ is nondenumerable, the determinant in the denominator cannot vanish. 
The polynomials in turn uniquely determine real numbers $a_{n}$ and $b_{n}, n \in$ $\{0,1,2, \ldots\}$, where $a_{0}=0$ and $a_{n}>0$ for $n>0$, such that $P_{n+1}(z)=\left(z-b_{n}\right) P_{n}(z)$ $-a_{n}^{2} P_{n-1}(z)$ (see, for example, [Ak, C]). Such recursion relations have been studied in [BGH1, 2, 3, 7; BMM1, 2; BM], mainly for cases where $R(z)$ is a polynomial. The reason for interest in such three-term recursion relations is that they fix a halfinfinite Jacobi matrix

$$
\mathscr{J}=\left(\begin{array}{ccccc}
b_{0} & a_{1} & 0 & 0 & \cdots \\
a_{1} & b_{1} & a_{2} & 0 & \ldots \\
0 & a_{2} & b_{2} & a_{3} & \ldots \\
0 & 0 & a_{3} & \ldots & \ldots
\end{array}\right)
$$

whose spectrum is related to $\mu$, as the following theorem shows.

THEOREM [BH]. Let $R(z)$ be of the form $(*)$ with $\left|b_{1}\right| \leqslant 1$, and let $J \subset \mathbf{R} \cup\{\infty\}$. Then the associated Jacobi matrix $\mathscr{J}$ is selfadjoint, with spectrum $J$ and spectral density $\mu$.

The last statement in the theorem asserts that

$$
\left\langle 0\left|(\mathscr{J}-z I)^{-1}\right| 0\right\rangle=\int_{J} \frac{d \mu(s)}{s-z}=F(z), \quad z \notin J,
$$

where $|j\rangle$ denotes the column vector with one in the $(j+1)$ th position and zeros elsewhere, $\langle j|$ is its transpose, $I$ is the identity matrix on $l_{2}$ and $F(z)$ is called the spectral function associated with $\mathscr{J}$.

It is proved in [BBM] that $b_{j}=0(j=0,1,2, \ldots)$ for $R(z)=z^{2}-\lambda$ with $\lambda>3$, while the sequence $\left\{a_{j}\right\}_{j=1}^{\infty}$ is limit periodic. Similar results are proved in [BGH7] for various other families of polynomial transformations including $R(z)=\alpha^{N+1} T_{N}(z / \alpha)$ for $\alpha>2$, where $T_{N}(z)$ is the Chebychev polynomial of degree $N$.

Functional equations. We derive the functional equation associated with the spectral function $F(z)$, the moment generating function $g(z)$ and the potential function $H(z)$ in the case where $R(z)$ is given by $(*)$ with $\left|b_{1}\right| \leqslant 1$.

For $z \notin J$, using (2) we have

$$
\begin{aligned}
F(z) & =\int \frac{d \mu(s)}{z-s}=\frac{1}{N} \int \sum_{i=1}^{N} \frac{1}{z-R_{i}^{-1}(s)} d \mu(s) \\
& =\frac{1}{N} \int \frac{P^{\prime}(z)-Q^{\prime}(z) s}{P(z)-Q(z) s} d \mu(s) \\
& =\frac{1}{N} \frac{P^{\prime}(z)}{Q(z)} F(R(z))-\frac{1}{N} \frac{Q^{\prime}(z)}{Q(z)} \int \frac{s}{R(z)-s} d \mu(s) \\
& =\frac{1}{N} \frac{P^{\prime}(z)}{Q(z)} F(R(z))-\frac{1}{N} \frac{Q^{\prime}(z)}{Q(z)}\{R(z) F(R(z))-1\} .
\end{aligned}
$$


The functional equation obeyed by the spectral function is thus

$$
F(z)=\frac{1}{N}\left\{R^{\prime}(z) F(R(z))+\frac{Q^{\prime}(z)}{Q(z)}\right\} .
$$

The moment generating function is defined by

$$
g(z)=\int_{J} \frac{d \mu(s)}{1-z s} \quad\left(=\sum_{n=0}^{\infty} M_{n} z^{n}\right),
$$

and, since $g(z)=(1 / z) F(1 / z)$, it follows from (9) that

$$
g(z)=\frac{1}{N}\left\{\frac{R^{\prime}(1 / z)}{z R(1 / z)} g\left(\frac{1}{R(1 / z)}\right)+\frac{Q^{\prime}(1 / z)}{z Q(1 / z)}\right\} .
$$

An alternative method to the one described above for calculating the moments of $\mu$ (when they exist) is to substitute the possibly formal series $g(z)=\sum_{n=0}^{\infty} M_{n} z^{n}$ into both sides of the latter equation and equate coefficients of powers of $z$.

The potential function associated with $\mu$ is defined by

$$
H(z)=\int_{J} \ln (z-s) d \mu(s)
$$

when it is possible to specify the branch of the ln which is taken as one passes "about" the set $J$; see [BGM]. $H(z)$ can be well defined, for example, when $J \subset \mathbf{R}$ or when $R(z)$ is a polynomial, so that all of $J$ is accessible from $\infty$. In such cases, by integrating (9) and choosing the integration constant so that $H(z) \sim \ln z$ near $\infty$, we find

$$
H(z)=\frac{1}{N}\left\{H(R(z))+\ln \left(\frac{Q(z)}{b}\right)\right\}
$$

where $b$ is the coefficient of the highest power of $z$ in $Q(z)$. We remark that, when $R(z)$ is a polynomial, $\exp (H(z))$ solves the Böttcher functional equation associated with $R(z)$ (see [BGH5, BGM]).

Functional equations of the above forms occur in statistical physics [BGM, DDI, DEE, DH]: one is able to tie in solutions of such equations with the theory of balanced measures on Julia sets.

\section{Condensed Julia sets.}

The example $z \rightarrow\left(z^{3}-\lambda z\right) / z$ compared with $z \rightarrow\left(z^{2}-\lambda\right)$ for $\lambda>2$. We consider what happens in the limit as $\varepsilon \rightarrow 0$ to the Julia set $J_{\varepsilon}$ and balanced measure $\mu_{\varepsilon}$ for the rational transformation

$$
R_{\varepsilon}(z)=z^{2}-\lambda+\varepsilon / z, \quad \lambda>2 .
$$

We begin by recalling the construction of the Julia set $J$ and balanced measure $\mu$ for $R(z)=z^{2}-\lambda . J$ is the limit of the decreasing sequence of sets $J^{(n)}$ constructed as follows. $J^{(0)}=I^{(0)}$, where $I^{(0)}=[-a, a]$ with $a=1 / 2+\sqrt{\lambda+1 / 4}$, this being the largest fixed point of $R$. Then we define inductively $J^{(n+1)}=R^{-1} J^{(n)}$, and it is readily shown that $J^{(n+1)} \subset J^{(n)}$ because $J^{(1)} \subset J^{(0)}$. We find $J^{(1)}=I_{-}^{(1)} \cup I_{+}^{(1)}$, where

$$
I_{-}^{(1)}=[-\sqrt{\lambda+\sqrt{a}},-\sqrt{\lambda-\sqrt{a}}] \text { and } I_{+}^{(1)}=[+\sqrt{\lambda-\sqrt{a}},+\sqrt{\lambda+\sqrt{a}}]
$$




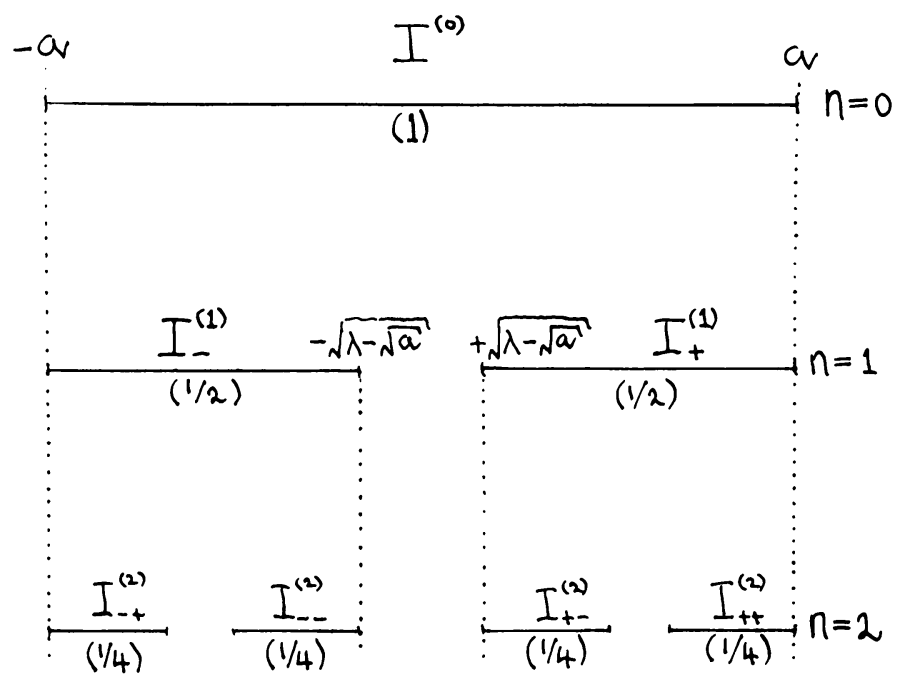

FIGURE 1 . The successive generations of intervals approximating the Julia set for $z^{2}-\lambda$ with $\lambda>2$. The numbers in parentheses give the balanced measure of the corresponding intervals.

are disjoint intervals, the preimages under the two inverse functions $R_{+}^{-1}(z)=$ $\pm \sqrt{\lambda+z}$ of $I^{(0)}$. Similarly we find $J^{(n)}$ consists of $2^{n}$ disjoint intervals $I_{-++}^{(n)} \ldots++$, $I_{-++}^{(n)} \ldots+, \ldots, I_{+++}^{(n)} \ldots++$, these being labelled according to the sequence of applications of $R_{ \pm}^{-1}(z)$ used to obtain them. For example $I_{++-}^{(3)}=R_{+}^{-1}\left(R_{+}^{-1}\left(R_{-}^{-1}\left(I^{(0)}\right)\right)\right)$. The end points of the intervals at the $n$th step consist of the set of all points

$$
\pm \sqrt{\lambda \pm \sqrt{\lambda \pm \cdots \pm \sqrt{a}}}
$$

where there are $(n+1)$ plus-or-minus signs. The relationship between successive sets of intervals is illustrated in Figure 1. Finally, $J=\bigcap_{n=1}^{\infty} J^{(n)}$, which is a generalized Cantor set of Lebesgue measure zero [Br], and which can be considered as the set of points $\{ \pm \sqrt{(\lambda} \pm \sqrt{(\lambda \pm} \cdots\}$ where all infinite sequences of plus and minus signs are included.

A sequence of measures $\left\{\mu^{(n)}\right\}_{n=0}^{\infty}$ which converge weakly to $\mu$ can be constructed as described following (2). $\mu^{(0)}$ associates total mass 1 with $I^{(0)}, \mu^{(1)}$ associates total mass $1 / 2$ with each of the intervals $I_{ \pm}^{(1)}, \ldots$, and $\mu^{(n)}$ associates equal mass $1 / 2^{n}$ with each of the $2^{n}$ intervals that constitute $J^{(n)}$. Notice that since successive generations of intervals are nested we have

$$
\mu\left(I_{ \pm \pm \pm \cdots \pm}^{(n)}\right)=\mu^{(m)}\left(I_{ \pm \pm \pm \cdots \pm}^{(n)}\right)=1 / 2^{n}
$$

when $m \geqslant n$.

We now describe the construction of $J_{\varepsilon}$ and $\mu_{\varepsilon}$ for $R_{\varepsilon}(z)=z^{2}-\lambda+\varepsilon / z$ when $0<\varepsilon \ll 1$; see Figure 2. Let $I^{(0)}=[-a-\delta, a+\delta]$, where $a+\delta$ is the positive fixed point of $R_{\varepsilon}(z)$, so $\delta \rightarrow 0$ as $\varepsilon \rightarrow 0$. $J_{\varepsilon}$ is contained in $I^{(0)}$ since $R_{\varepsilon}^{-1} I^{(0)} \subset I^{(0)}$. Let $J^{(n+1)}=R_{\varepsilon}^{-1} J^{(n)}$ so that $J^{(n+1)} \subset J^{(n)}$ and $J_{\varepsilon}=\bigcap_{n=1}^{\infty} J^{(n)}$ is a triadic Cantor set of Lebesgue measure zero. Then $J^{(1)}=I_{-}^{(1)} \cup I_{0}^{(1)} \cup I_{+}^{(1)}$, where $I_{ \pm}^{(1)}$ are what they 


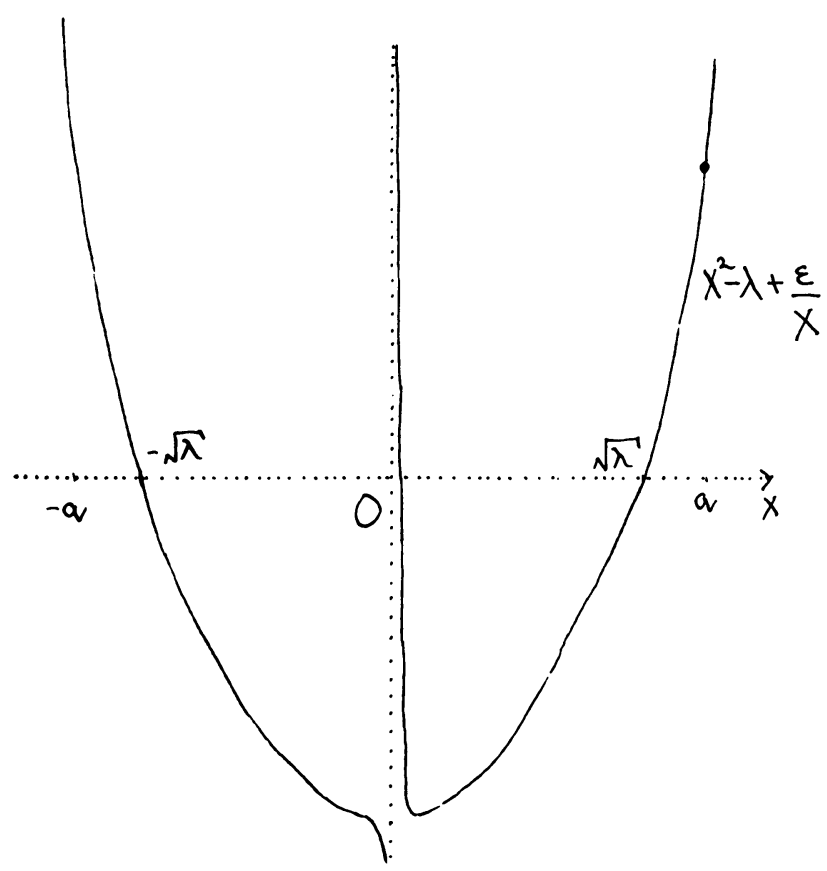

FIgURE 2. $R_{\varepsilon}(x)$ for $\lambda>2$ and $0<\varepsilon \ll 1$. The Julia set $J_{\varepsilon}$ is contained approximately in $[-a, a]$ and can be obtained by inverse iteration.

were above, to order $\varepsilon$, and $I_{0}^{(1)}$ is an interval located within a distance of order $\varepsilon$ from the origin $O$, whose length is of order $\varepsilon$. We denote the branches of the inverse of $R$ by $R_{ \pm}^{-1}(z)$ and $R_{0}^{-1}(z)$, where $R_{ \pm}^{-1}(z)$ are approximately what they were above. At the $n$th step we obtain $3^{n}$ disjoint closed intervals, which we label in terms of the sequence of inverse branches by which they were obtained, according to

$$
I_{\alpha \beta \cdots \gamma}^{(n)}=R_{\alpha}^{-1}\left(R_{\beta}^{-1}\left(\cdots R_{\gamma}^{-1}\left(I^{(0)}\right) \cdots\right)\right)
$$

for any sequence of $n$ symbols $\alpha, \beta, \ldots, \gamma \in\{+, 0,-\}$. We call $\alpha \beta \cdots \gamma$ the code of $I_{\alpha \beta \cdots \gamma}^{(n)}$. Notice that any interval whose code contains a zero is of length of order $\varepsilon$, uniformly over all codes. (The uniformity follows from the hyperbolicity of $J$ for $z^{2}-\lambda$ when $\lambda>2$.) Notice also that if $I_{\alpha \beta \cdots \gamma \delta}^{(n)}$ has $\delta=0$ while $\alpha, \beta, \ldots, \gamma \in\{+,-\}$, then $I_{\alpha \beta \ldots \gamma \delta}^{(n)}$ is located within a distance of order $\varepsilon$ from $R_{\alpha}^{-1}\left(R_{\beta}^{-1}\left(\cdots R_{\gamma}^{-1}(0) \cdots\right)\right)$, uniformly over all codes. Finally notice that if $I_{\alpha \beta \cdots \gamma}^{(n)}$ and $I_{\alpha \beta \cdots \gamma \cdots \delta}^{(m)}$ have in common the initial sequence $\alpha \beta \cdots \gamma$, where $n \leqslant m$, then the latter interval is contained in the former.

We construct a sequence of measures $\left\{\mu_{\varepsilon}^{(n)}\right\}$, converging weakly to the balanced measure $\mu_{\varepsilon}$, as before. $\mu_{\varepsilon}^{(n)}$ associates mass $1 / 3^{n}$ with each of the $3^{n}$ obtained at the $n$th step. In particular, we discover $\mu_{\varepsilon}\left(I_{\alpha \beta \cdots \gamma \delta}^{(n)}\right)=1 / 3^{n}$, where we suppose $\delta=0$ and $\alpha, \beta, \ldots, \gamma \in\{+,-\}$, so that this mass is concentrated within a distance of order $\varepsilon$ from $R_{\alpha}^{-1}\left(R_{\beta}^{-1}\left(\cdots R_{\gamma}^{-1}(0) \cdots\right)\right)$, see Figure 3.

We now take the limit of $J_{\varepsilon}$ as $\varepsilon \rightarrow 0$. Since all intervals in $J^{(n)}$ whose codes contain zeros shrink to points, we readily find that $C=\lim _{\varepsilon \rightarrow 0} J_{\varepsilon}$ consists of the Julia set $J$ associated with $z^{2}-\lambda$, together with the origin $O$ and all its preimages 


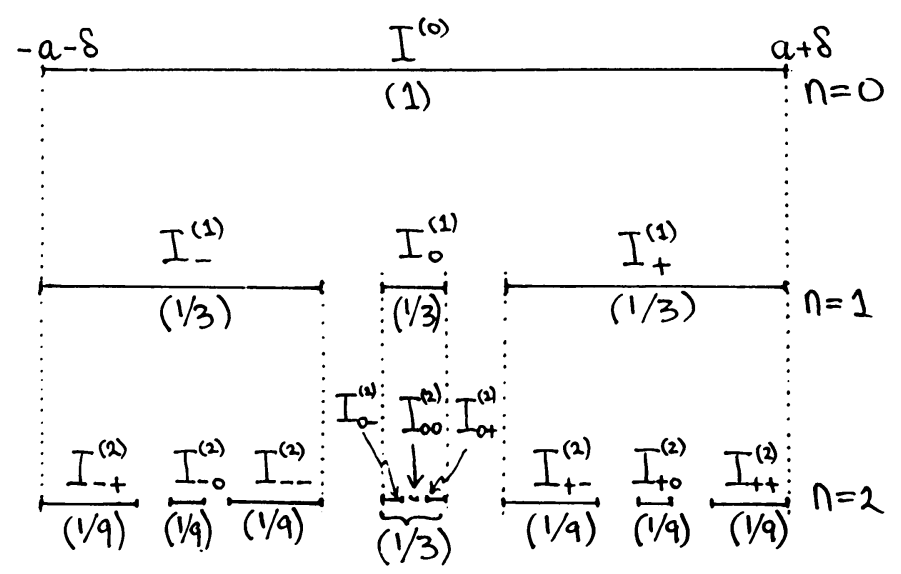

FIgURE 3. The successive generations of intervals approximating the Julia set for $z^{2}-\lambda+\varepsilon / z$. The numbers in parentheses give the balanced measures of the corresponding intervals.

under $R^{-n}$, where $R(z)=z^{2}-\lambda$. Thus, the condensed Julia set is $C=J \cup P$ with $P=\{ \pm \sqrt{(\lambda} \pm \sqrt{(\lambda} \pm \cdots \pm \sqrt{\lambda)}\}$, where all finite length chains are included. Thus $C$ is the same as the closure of $P$. If we think of $J$ as being obtained by starting from $[-a, a]$, taking out the "middle" interval

$$
(-\sqrt{\lambda-\sqrt{a}},+\sqrt{\lambda-\sqrt{a}})
$$

followed by taking out the "middles"

$$
(-\sqrt{\lambda+\sqrt{\lambda-\sqrt{\lambda}}},-\sqrt{\lambda-\sqrt{\lambda-\sqrt{\lambda}}}), \quad(+\sqrt{\lambda-\sqrt{\lambda-\sqrt{\lambda}}}, \sqrt{\lambda+\sqrt{\lambda-\sqrt{\lambda}}})
$$

of the remaining pair of intervals, and so on, thus mimicking the usual Cantor set construction, we see that $P$ consists of a single point lying in each one of the excluded intervals.

The limiting measure $\sigma$, to which $\mu_{\varepsilon}$ converges weakly as $\varepsilon \rightarrow 0$, is readily found. It is given by

$$
d \sigma(x)=\sum_{n=0}^{\infty} \sum_{m=1}^{2^{n}} \frac{1}{3^{n+1}} \delta\left(x-x_{m}^{(n)}\right) d x,
$$

where $\left\{x_{m}^{(n)}\right\}_{m=1}^{2^{\prime \prime}}$ denotes the preimages of order $n$ under $z^{2}-\lambda$ of $O$, and $\delta(x)$ denotes the Dirac delta function. We call $\sigma$ the condensed measure associated with $z \rightarrow\left(z^{3}-\lambda z\right) / z:$ it is illustrated in Figure 4.

$$
\sigma(P)=\sum_{n=0}^{\infty} \frac{2^{n}}{3^{n+1}}=1,
$$

whence $\sigma(J)=0$. For contrast, notice that $\mu(P)=0$ and $\mu(J)=1$, where $\mu$ is the balanced measure for $z^{2}-\lambda$. (We think of the condensation process as stealing measure from $J$.)

$\sigma$ is not a balanced measure, although it is a weak limit of balanced measures. If we define the inverse branches of $\left(z^{3}-\lambda z\right) / z$ for all $z$ by $R_{1}^{-1}(z)=-\sqrt{\lambda+z}$, 


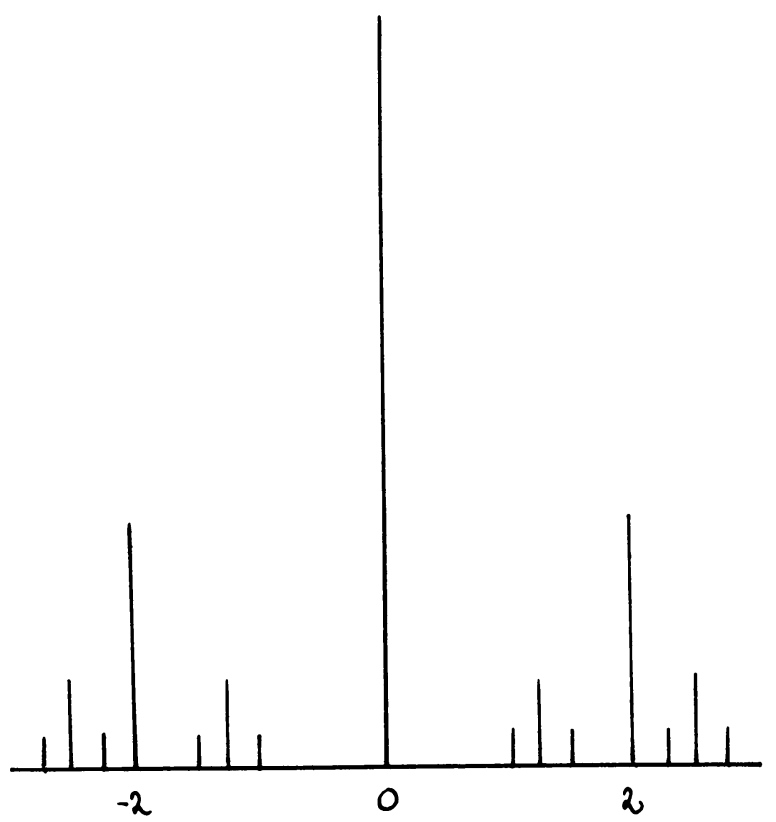

FIgURE 4. The condensed measure $\sigma$ for $\left(z^{3}-4 z\right) / z$. The height of the spike above $x$ shows the amount of measure at that point. This also represents the spectral density for an associated Jacobi matrix, $\tilde{\mathscr{J}}$.

$$
\begin{aligned}
& R_{2}^{-1}(z)=\sqrt{\lambda+z} \text { and } R_{3}^{-1}(z)=0 \text {, then } \\
& \sigma\left(R_{i}^{-1}(E)\right)=\frac{1}{3} \sigma(E)+\frac{1}{3} \chi_{E}(-\lambda), \quad i=1,2, \\
& \sigma\left(R_{3}^{-1}(E)\right)= \begin{cases}\frac{1}{3} & \text { if } E \neq \varnothing, \\
0 & \text { if } E=\varnothing,\end{cases}
\end{aligned}
$$

where $\chi_{E}(z)$ denotes the characteristic function of the Borel set $E \subset \hat{\mathbf{C}}$. On the other hand, the property (2) does remain true; namely

$$
\begin{aligned}
\int_{\hat{\mathbf{C}}} f(z) d \sigma(z) & =\frac{1}{3} \int_{\hat{\mathbf{C}}} \sum_{i=1}^{3} f\left(R_{i}^{-1}(z)\right) d \sigma(z) \\
& =\frac{1}{3} \int_{\hat{\mathbf{C}}} \sum_{i=1}^{2} f\left(R_{i}^{-1}(z)\right) d \sigma(z)+\frac{1}{3} f(0),
\end{aligned}
$$

as can be verified directly from (12).

Following similar lines to the derivation of (9), we find here, using (13), that the spectral function $F(z)=\int_{\hat{\mathbf{c}}} d \sigma(s) /(z-s)$ obeys the functional equation

$$
F(z)=\frac{2}{3} z \cdot F\left(z^{2}-\lambda\right)+\frac{1}{3} \cdot \frac{1}{z} \text {. }
$$

This is exactly the same as that which is obtained on taking $N=3$ and $R(z)=$ $\left(z^{3}-\lambda z\right) / z$, so $Q(z)=z$ in equation (9). (This equation contrasts with $F(z)=z$. $F\left(z^{2}-\lambda\right)$ which is obeyed by the spectral function associated with $R(z)=z^{2}-\lambda$.) Similarly the moment generating function $g(z)=\int_{\hat{\mathbf{C}}} d \sigma(s) /(1-s z)$ obeys

$$
g(z)=\frac{2}{3} \cdot \frac{1}{1-\lambda z^{2}} \cdot g\left(\frac{z^{2}}{1-\lambda z^{2}}\right)+\frac{1}{3},
$$


while the Green's function $H(z)=\int_{\hat{\mathbf{C}}} \ln (z-s) d \sigma(s)$ satisfies

$$
H(z)=\frac{1}{3} H\left(z^{2}-\lambda\right)+\frac{1}{3} \ln z .
$$

These equations could have been deduced directly from (9), (10) and (11), with $R(z)=z^{2}-\lambda+\varepsilon / z$, by taking weak limits when $\lambda>2$. However, their validity does not rest upon the limiting process, and they are true for the condensed measure defined by (12) for all values of $\lambda$, regardless of whether or not $J$ is hyperbolic.

We are also able to calculate the moments of $\sigma$ using the method described in $\$ 2$ (cf. equations (4)-(6)) provided that $R(z)$ is taken to be $\left(z^{3}-\lambda z\right) / z$ without cancellation. The reason is simply that the integration formula (13) holds, and the symmetric functions

$$
S_{m}(z)=\sum_{j=1}^{3}\left(R_{j}^{-1}(z)\right)^{m}
$$

are correctly given by the algorithm (4) with $z^{N}+a_{1} z^{N-1}+\cdots+a_{N}=z^{3}-\lambda z$ and $b_{1} z^{N-1}+b_{2} z^{N-2}+\cdots+b_{N}=z$.

In fact, both in the case $R(z)=\left(z^{3}-\lambda z\right) / z$ and in the case $R(z)=z^{2}-\lambda$ we find that, since the "condensation point" is 0 ,

$$
S_{2 n}(z)=2 \cdot(z+\lambda)^{n}, \quad S_{2 n-1}(z)=0, \quad n=1,2,3, \ldots,
$$

so that in the balanced case $R(z)=z^{2}-\lambda$ the moments $M_{n}=\int z^{n} d \mu$ are generated recursively using

$$
M_{2 n}=\frac{2}{2} \int(z+\lambda)^{n} d \mu, \quad M_{2 n-1}=0, \quad n=1,2, \ldots ;
$$

while in the condensed case $R(z)=\left(z^{3}-\lambda z\right) / z$ the moments $\tilde{M}_{n}=\int z^{n} d \sigma$ are generated recursively using

$$
\tilde{M}_{2 n}=\frac{2}{3} \int(z+\lambda)^{n} d \sigma, \quad \tilde{M}_{2 n-1}=0, \quad n=1,2, \ldots
$$

In Table 1 we compare the first six moments in the two cases.

TABLE 1

\begin{tabular}{|c|c|c|}
\hline$n$ & $M_{n}$ & $\tilde{M}_{n}$ \\
\hline 0 & 1 & 1 \\
1 & 0 & 0 \\
2 & $\lambda$ & $\frac{2}{3} \lambda$ \\
3 & 0 & 0 \\
4 & $\lambda \cdot(\lambda+1)$ & $\frac{2}{3} \lambda \cdot\left(\lambda+\frac{2}{3}\right)$ \\
5 & 0 & 0 \\
6 & $3 \lambda^{2}+\lambda^{3}$ & $\frac{4}{3} \lambda^{2}+\frac{2}{3} \lambda^{3}$ \\
\hline
\end{tabular}

First six moments $M_{n}$ of the balanced measure for $z^{2}-\lambda$ compared with the corresponding moments $M_{n}$ of the condensed measure for $\left(z^{3}-\lambda z\right) / z$. 
For $\lambda>2$, since the measure is symmetric about the origin, the Jacobi matrix associated with the three-term recurrence formula for the orthogonal polynomials is of the form

$$
\mathscr{J}=\left(\begin{array}{cccccc}
0 & a_{1} & 0 & 0 & 0 & \ldots \\
a_{1} & 0 & a_{2} & 0 & 0 & \ldots \\
0 & a_{2} & 0 & a_{3} & 0 & \ldots \\
0 & 0 & a_{3} & 0 & a_{4} & \ldots \\
0 & 0 & 0 & a_{4} & 0 & \ldots \\
. & . & . & . & . & \ldots
\end{array}\right)
$$

both in the balanced case, $R(z)=z^{2}-\lambda$, and in the condensed case, $R(z)=$ $\left(z^{3}-\lambda z\right) / z$. Denoting the Jacobi matrices in these two cases by $\mathscr{J}$ and $\tilde{\mathscr{J}}$ respectively, and using the identities $M_{n}=\left\langle 0\left|\mathscr{J}^{n}\right| 0\right\rangle$ and $\tilde{M}_{n}=\left\langle 0\left|\tilde{J}^{n}\right| 0\right\rangle$, we obtain from (14) and (15), respectively,

$$
\left\langle 0\left|\mathscr{J}^{2 n}\right| 0\right\rangle=\frac{2}{2}\left\langle 0\left|(\mathscr{J}+\lambda I)^{n}\right| 0\right\rangle, \quad n=1,2,3, \ldots,
$$

and

$$
\left\langle 0\left|\tilde{J}^{2 n}\right| 0\right\rangle=\frac{2}{3}\left\langle 0\left|(\tilde{\mathscr{J}}+\lambda I)^{n}\right| 0\right\rangle, \quad n=1,2,3, \ldots
$$

We now derive from (16) the renormalization equation obeyed by $\mathscr{J}$ first obtained by [BBM], from which can be deduced the recursion relations among the $a_{n}$ 's. ([BBM] shows that the latter implies that $\left\{a_{n}\right\}_{n=1}^{\infty}$ is a limit periodic sequence for $\lambda>3$.) We then contrast this with what happens when the same approach is followed, starting from (17), to get a relation between $\tilde{\mathscr{J}}$ and a transform of it.

Let $D$ denote the decimation operator defined by $D^{*}|n\rangle=|2 n\rangle$, where * denotes the adjoint, so that

$$
D=\left(\begin{array}{cccccc}
1 & 0 & 0 & 0 & .0 & \ldots \\
0 & 0 & 1 & 0 & 0 & \ldots \\
0 & 0 & 0 & 0 & 1 & \ldots \\
. & . & . & . & . & \ldots
\end{array}\right)
$$

Notice that $D D^{*}=I$ while

$$
D^{*} D=\left(\begin{array}{ccccccc}
1 & 0 & 0 & 0 & 0 & 0 & \ldots \\
0 & 0 & 0 & 0 & 0 & 0 & \ldots \\
0 & 0 & 1 & 0 & 0 & 0 & \ldots \\
0 & 0 & 0 & 0 & 0 & 0 & \ldots \\
0 & 0 & 0 & 0 & 1 & 0 & \ldots \\
. & . & . & . & . & . & \ldots
\end{array}\right) .
$$

Then it is readily proved inductively that for $\mathscr{J}$ of the form above (with zero diagonal)

$$
\left\langle 0\left|\left(D \mathscr{J}^{2} D^{*}\right)^{n}\right| 0\right\rangle=\left\langle 0\left|\mathscr{J}^{2 n}\right| 0\right\rangle
$$

Using this in (16), we obtain

$$
\left\langle 0\left|\left(D \mathscr{J}^{2} D^{*}\right)^{n}\right| 0\right\rangle=\left\langle 0\left|(\mathscr{J}+\lambda I)^{n}\right| 0\right\rangle, \quad n=0,1,2, \ldots,
$$




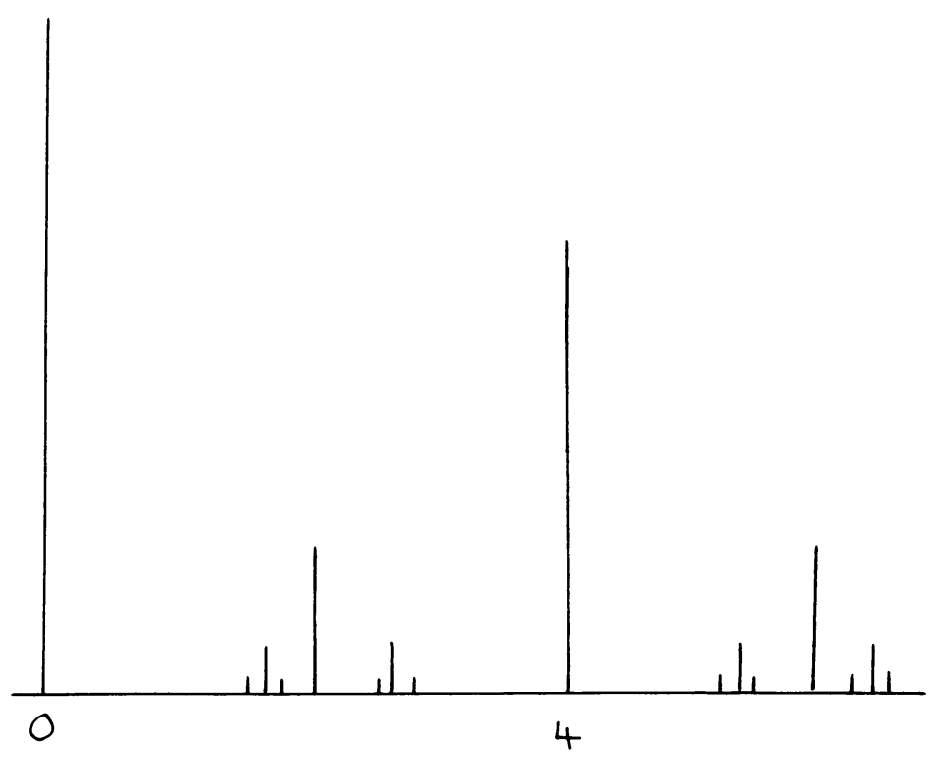

Figure 5. The spectral density for the tridiagonal matrix $D \tilde{\mathscr{J}}^{2} D^{*}$; see text.

from which it follows, since both $D \mathscr{J}^{2} D^{*}$ and $(\mathscr{J}+\lambda I)$ are tridiagonal, that they are identical. That is,

$$
\begin{aligned}
\left(\begin{array}{cccc}
a_{0}^{2}+a_{1}^{2} & a_{1} a_{2} & 0 & \ldots \\
a_{1} a_{2} & a_{2}^{2}+a_{3}^{2} & a_{3} a_{4} & \ldots \\
0 & a_{3} a_{4} & a_{4}^{2}+a_{5}^{2} & \ldots \\
0 & 0 & a_{5} a_{6} & \ldots \\
\cdot & \cdot & \cdot & \ldots
\end{array}\right) & =D \mathscr{J}^{2} D^{*}=(\mathscr{J}+\lambda I) \\
& =\left(\begin{array}{cccc}
\lambda & a_{1} & 0 & \ldots \\
a_{1} & \lambda & a_{2} & \ldots \\
0 & a_{2} & \lambda & \ldots \\
0 & 0 & a_{3} & \ldots \\
\cdot & \cdot & \cdot & \ldots
\end{array}\right),
\end{aligned}
$$

where $a_{0}=0$.

We remark that the latter implies through straightforward algebra the identity $D\left(\mathscr{J}^{2}-\lambda I\right)=\mathscr{J} D$, which was obtained in a different way in [BBM].

We now look at what happens for $\tilde{\mathscr{J}}$. From (17), following the same line, we find

$$
\left\langle 0\left|\left(D \tilde{J}^{2} D^{*}\right)^{n}\right| 0\right\rangle=\frac{2}{3}\left\langle 0\left|(\tilde{\mathscr{J}}+\lambda I)^{n}\right| 0\right\rangle, \quad n=1,2,3, \ldots
$$

Although both $D \tilde{\mathscr{J}}^{2} D^{*}$ and $(\tilde{\mathscr{J}}+\lambda I)$ are tridiagonal, this equation does not allow us to deduce a simple relation between the two operators, in contrast to the previous case. All we can deduce is that the spectral density of $D \mathscr{J}^{2} D^{*}$ is $2 / 3$ times that of $\mathscr{J}+\lambda I$ plus a point mass of $1 / 3$ at the origin; see Figure 5 .

Equation (18) can be used to calculate the coefficients $\tilde{a}_{n}$ occurring in $\tilde{\mathscr{J}}$. Some computed values are given in Table 2 , together with corresponding values $a_{n}$ for $z^{2}-\lambda$, when $\lambda=2$. 
TABLE 2

\begin{tabular}{|l|r|r|}
\hline$n$ & $a_{n}^{2}$ & $\tilde{a}_{n}^{2}$ \\
\hline 1 & 2 & $4 / 3$ \\
2 & 1 & $4 / 3$ \\
3 & 1 & $2 / 3$ \\
4 & 1 & $5 / 3$ \\
5 & 1 & $3 / 5$ \\
6 & 1 & $16 / 15$ \\
7 & 1 & $5 / 6$ \\
8 & 1 & $7 / 4$ \\
9 & 1 & .535 \\
10 & 1 & 1.01 \\
\hline
\end{tabular}

\begin{abstract}
Recurrence coefficients $\tilde{a}_{n}$ associated with the condensed measure for $\left(z^{3}-2 z\right) / z$. The spectrum of $\tilde{g}_{\text {in }}$ this case is $[-2,2]$, which contains a dense forest of eigenvalues. The $a_{n}$ 's are the corresponding values for the balanced measure $d \mu(x)=\pi^{-1} \cdot\left(2-x^{2}\right)^{-1 / 2}, x \in[-2,2]$, associated with $z^{2}-2$. The asymptotic behavior of the $\tilde{a}_{n}$ 's is an open question.
\end{abstract}

Condensed Julia sets and condensed measures in general. The procedure described for the example $z \rightarrow z^{2}-\lambda+\varepsilon / z$ is readily generalized in several ways. Let $R(z)=P(z) / Q(z)$ denote any rational transformation of degree $N>1$, where there are no common factors between the polynomials $P$ and $Q$. Then a condensed Julia set $C$ and a condensed measure $\sigma$ may be associated with the unreduced rational fraction

$$
R(z) \cdot \prod_{i=1}^{k}\left(z-a_{i}\right)^{\gamma_{i}} / \prod_{i=1}^{k}\left(z-a_{i}\right)^{\gamma_{i}},
$$

where the $\gamma_{i}$ 's and $k$ are finite positive integers, and $a_{i} \in \mathbf{C}$ for $i=1,2, \ldots, k$. We assume that none of the $a_{i}$ 's is an exceptional point, in the nomenclature of Brolin [Br]. Then we define $C$ to be the closure of the set $P$ of all preimages of finite order under $R$ of the $a_{i}$; and, because of the last assumption, $C=P \cup J$, where $J$ is the Julia set for $R(z)$. We call $a_{i}$ a condensation point of order $\gamma_{i}$, and we call $R$ the generator of the condensed Julia set.

The condensed measure associates mass with the set $P$ as follows. Let $M=\sum_{s=1}^{k} \gamma_{s}$, and let an inverse image of order $n$ under $R$ of $a_{s}$ be denoted by

$$
R_{i}^{-1}\left(R_{j}^{-1} \cdots R_{m}^{-1}\left(a_{s}\right) \cdots\right)=a_{s}^{i j \cdots m(n)}
$$

where $\left\{R_{i}^{-1}(z)\right\}_{i=1}^{N}$ is a complete assignment of branches of $R^{-1}$. When $n=0$, take this expression to mean just $a_{s}$. Assuming that

$$
P=\bigcup_{\substack{s \\ i j \cdots m(n)}} a_{s}^{i j \cdots m(n)}
$$


contains no cycles or branch points of $R^{-1}$, we define $\sigma$ by

$$
\sigma\left(a_{s}^{i j \cdots m(n)}\right)=\gamma_{s} /(M+N)^{n+1} \text {. }
$$

In the case where $P$ contains branch points or cycles, the total mass attached to the "coalesced points" is summed. Finally, $\sigma$ assigns no measure to any set $E$ such that $E \cap P=\varnothing . \sigma$ is normalized since

$$
\sigma(\hat{\mathbf{C}})=\sigma(P)=\sum_{s=1}^{k} \sum_{n=0}^{\infty} \frac{\gamma_{s} N^{n}}{(M+N)^{n+1}}=\frac{M}{M+N} \sum_{n=0}^{\infty}\left(\frac{N}{M+N}\right)^{n}=1 .
$$

Under appropriate conditions one can prove that $\sigma$ and $C$ are related by "a condensation process' associated with the limit as $\varepsilon \rightarrow 0$ of the family of rational transformations

$$
R(z, \varepsilon)=R(z)+\varepsilon \sum_{s=1}^{k}\left(z-a_{d}\right)^{-\gamma_{s}},
$$

as the following theorem shows.

THEOREM. Let $R(z)$ be a rational transformation of degree $N$ with hyperbolic Julia set $J$, and let $\left\{a_{i}\right\}_{i=1}^{M}$ be condensation points of order one, with $a_{i} \notin J$ for $i=1,2, \ldots, M$, where no $a_{i}$ is a critical point of $R(z)$, and no $a_{i}$ belongs to a $k$-cycle of $R(z)$ for any finite $k$. Let $J_{\varepsilon}$ and $\mu_{\varepsilon}$ denote respectively the Julia set and balanced measure associated with

$$
R(z, \varepsilon)=R(z)+\varepsilon \sum_{s=1}^{M}\left(z-a_{s}\right)^{-1},
$$

and let $C$ and $\sigma$ denote respectively the condensed Julia set and condensed measure for the unreduced rational fraction

$$
R(z) \cdot \prod_{s=1}^{M}\left(z-a_{s}\right) / \prod_{s=1}^{M}\left(z-a_{s}\right) .
$$

Then $\lim _{\varepsilon \rightarrow 0} J_{\varepsilon}=C$ and, for any $f \in \mathscr{C}(\hat{\mathbf{C}})$,

$$
\lim _{\varepsilon \rightarrow 0} \int f(z) d \mu_{\varepsilon}(z)=\int f(z) d \sigma
$$

Proof. We prove the theorem in the special case $R(z, \varepsilon)=R(z)+\varepsilon / z$, where $\left|R^{\prime}(z)\right| \geqslant k>1$ for all $z \in J, 0 \notin J, R^{\prime}(0) \neq 0$, and the origin $O$ is not a $k$-cycle of $R$ for an finite $k$. Let $\left\{R_{j}^{-1}(z)\right\}_{j=1}^{N}$ be any complete assignment of branches of $R^{-1}$, and let $\left\{R_{j}^{-1}(z, \varepsilon)\right\}_{j=1}^{N+1}$ denote a corresponding assignment of branches of the inverse of $R(z, \varepsilon)$, chosen so that $R_{j}^{-1}(z, \varepsilon) \rightarrow R_{j}^{-1}(z)$ for $j=1,2, \ldots, N$, uniformly on any closed set which excludes all of the critical values of $R(z, \varepsilon)$ for all $\varepsilon$ sufficiently small. The branch $R_{N+1}^{-1}(z, \varepsilon)$ is discussed below.

In order to relate $J_{\varepsilon}$ and $\mu_{\varepsilon}$ to $C$ and $\sigma$, we seek a nonempty open set $M_{\varepsilon}$ lying close to $J_{\varepsilon}$, such that $R^{-1}\left(M_{\varepsilon}, \varepsilon\right) \subset M_{\varepsilon}$. The latter condition ensures $J_{\varepsilon} \subset M_{\varepsilon}$. By construction, $M_{\varepsilon}$ also lies close to $C=J \cup P$, where $P$ is the origin together with its preimages under $R$. 
Since $\left|R^{\prime}(z)\right| \geqslant k>1$ on $J$, it follows that for all sufficiently small $\varepsilon>0$, there is a neighborhood $N_{\delta}$ of $J$ such that $R_{i}^{-1}\left(N_{\delta}, \tilde{\varepsilon}\right) \subset N_{\delta}$ for $i=1,2, \ldots, N$, for all $0<\tilde{\varepsilon}<\varepsilon$, where $\delta \rightarrow 0$ as $\varepsilon \rightarrow 0$, and where $d\left(N_{\delta}, J\right)<\varepsilon$. Here $d(A, B)$ denotes the Hausdorff metric which measures the distance between subsets of $A$ and $B$ of $\hat{\mathbf{C}}$ according to

$$
d(A, B)=\underset{a \in A}{\operatorname{Max}} \operatorname{Min}_{b \in B} d(a, b)+\operatorname{Max}_{b \in B} \operatorname{Min}_{a \in A} d(a, b),
$$

where $d(a, b)$ is the distance between points $a$ and $b$ on $\hat{\mathbf{C}}$ (Poincaré metric). (We remark that by inverse iterating $N_{\delta}$ under all possible chains of inverses made from composing $R_{j}^{-1}(z, \varepsilon)$ for $j=1,2, \ldots, N$, one obtains a decreasing family of sets which converges to the "restricted inverse Julia set" $\bar{J}_{\varepsilon}$, which itself converges to $J$ as $\varepsilon \rightarrow 0$.)

Let $C_{\varepsilon}$ denote a circle of center $O$ and radius $\sqrt{\varepsilon} . \varepsilon$ is assumed to be so small that $C_{\varepsilon}$ contains no attractive $k$-cycle of $R(z, \varepsilon)$ for any $k$. Then $R\left(C_{\varepsilon}, \varepsilon\right)$ is readily shown to be contained in the complement of a circle $S_{\varepsilon}$ whose center is $R(0)$ and whose radius is of order $\sqrt{\varepsilon}$. Since $O \notin J$ we can ensure that $S_{\varepsilon}$ does not intersect $N_{\varepsilon}$ by choosing $\varepsilon$ small enough. It follows that

$$
R_{N+1}^{-1}\left(\hat{\mathbf{C}} \backslash S_{\varepsilon}, \varepsilon\right) \subset C_{\varepsilon} \text {. }
$$

We now construct $M_{\varepsilon}$ by taking the union of $N_{\varepsilon}$ with $C_{\varepsilon}$ and all of the preimages of all orders of $C_{\varepsilon}$ under $R(z, \varepsilon)$. If we define

$$
C_{\varepsilon}^{i j \cdots k}=R_{i}^{-1}\left(R_{j}^{-1} \cdots R_{k}^{-1}\left(C_{\varepsilon}\right) \cdots\right), \quad C_{\varepsilon}^{\varnothing}=C_{\varepsilon},
$$

for $i, j, \ldots, k \in\{1,2, \ldots, N+1\}$, these being inverse branches of $R(z, \varepsilon)$, then

$$
M_{\varepsilon}=N_{\delta} \cup \bigcup_{i, j, \ldots, k} C_{\varepsilon}^{i j \cdots k} .
$$

Now notice that the inverse images of all orders under $R(z)$ of $O$ stay strictly away from $R(0)$ (otherwise $O$ would have to belong to a $k$-cycle of $R(z)$ ), whence by continuity and using the fact that $J$ is hyperbolic for $R$, we must have

$$
\left\{\bigcup_{i, j, \ldots, k \in T} C_{\varepsilon}^{i j \cdots k}\right\} \cap S_{\varepsilon}=\varnothing,
$$

where $T=\{i, j, \ldots, k: i \neq N+1, j \neq N+1, \ldots, k \neq N+1\}$ when $\varepsilon$ is sufficiently small. From this together with (19) it follows that any $C_{\varepsilon}^{i j \cdots k}$, whose code $i j \cdots k$ contains an $(N+1)$ in the $l$ th position, is contained in the $C_{\varepsilon}^{i j \cdots k}$ whose code is obtained by keeping only the first $(l-1)$ symbols. Consequently,

$$
\bigcup_{i j \cdots k \in T} C_{\varepsilon}^{i j \cdots k}=\bigcup_{i j \cdots k} C_{\varepsilon}^{i j \cdots k}
$$

when $\varepsilon$ is sufficiently small.

Also observe from the hyperbolicity of $J$ with respect to $R$ that $C_{\varepsilon}^{i j \cdots k} \subset N_{\delta}$ when $i j \cdots k \in T$ and the code is sufficiently long. (The requisite length will increase with decreasing $\varepsilon$.) It follows that

$$
M_{\varepsilon}=N_{\delta} \cup \underset{\text { finitely many } i j \cdots k \in T}{\bigcup_{\varepsilon}^{i j \cdots k}}
$$

and that $R^{-1}\left(M_{\varepsilon}, \varepsilon\right) \subset M_{\varepsilon}$ for all sufficiently small $\varepsilon$. It follows that $J_{\varepsilon} \subset M_{\varepsilon}$. 


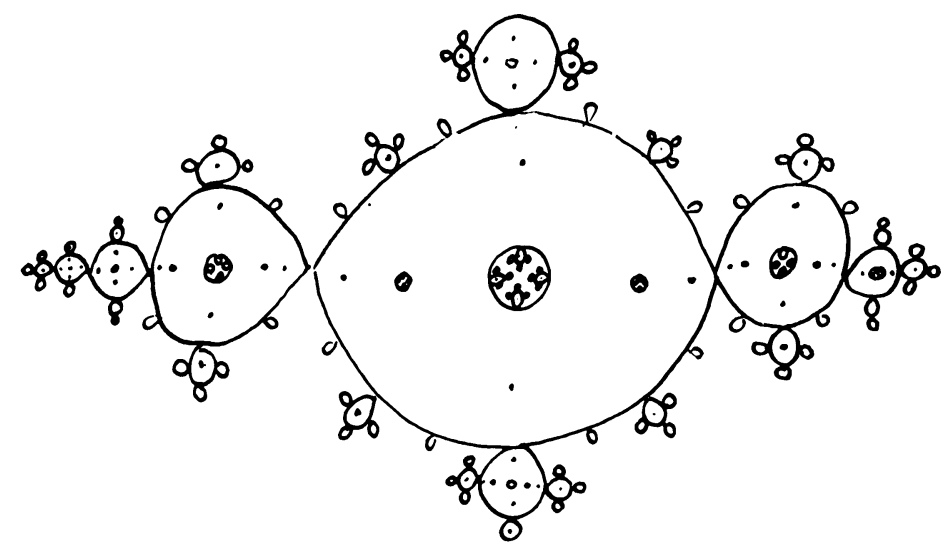

FIGURE 6. Qualitative picture of the Julia set for $z \rightarrow z^{2}-1+\varepsilon / z$ for small $\varepsilon>0$.

Now notice that $C_{\varepsilon}^{i, j \ldots k}$ has a diameter of order $\sqrt{\varepsilon}$, uniformly over all codes in $T$. Hence $d\left(M_{\varepsilon}, J_{\varepsilon}\right) \rightarrow 0$ as $\varepsilon \rightarrow 0$. Also notice that

$$
d\left(R_{i}^{-1}\left(R_{j}^{-1} \cdots R_{k}^{-1}(0) \cdots\right), C_{\varepsilon}^{i j \cdots k}\right)
$$

is of order $\sqrt{\varepsilon}$, where inverse branches of $R(z)$ are used, uniformly over any finite set of codes in $T$. Hence $D\left(M_{\varepsilon}, C\right) \rightarrow 0$ as $\varepsilon \rightarrow 0$. We now have $d\left(J_{\varepsilon}, C\right) \leqslant d\left(J_{\varepsilon}, M_{\varepsilon}\right)+$ $d\left(M_{\varepsilon}, C\right)$, where the right-hand side goes to zero with $\varepsilon$. This proves $\lim _{\varepsilon \rightarrow 0} J_{\varepsilon}=C$.

To prove the weak convergence it suffices to prove $\mu_{\varepsilon}(E) \rightarrow \sigma(E)$ for any open set $E \subset \hat{\mathbf{C}}$. Since $\mu_{\varepsilon}\left(N_{\varepsilon}\right) \rightarrow 0$ as $\varepsilon \rightarrow 0$ and $\delta \rightarrow 0$ and $\sigma(J)=0$, it suffices to suppose $J \cap E=\varnothing$. It then follows that $|P \cap E|$ is finite. Let $S$ denote the set of codes corresponding to the inverse images of $O$ under $\left\{R_{j}^{-1}(z)\right\}_{j=1}^{N}$. Choose $\varepsilon$ so small that $C_{\tilde{\varepsilon}}^{i j \cdots k} \subset E$ for all $i j \cdots k \in S$, for all $\tilde{\varepsilon} \leqslant \varepsilon$. Then $\mu_{\varepsilon}(E)=\sigma(E)$ for all $\tilde{\varepsilon}<\varepsilon$. Q.E.D.

In Figure 6, we sketch $J_{\varepsilon}$ for $z^{2}-z+\varepsilon / z$.

It has been proved by Demko [D] under much more general conditions, that one has a weak convergence theorem of the type given above. In such cases, it follows that an algorithm for the calculation of the moments of $\sigma$ (assuming that they exist - see below), together with the functional equations obeyed by the spectral function, the moment generating function and, the potential function, are correctly given by taking limits as $\varepsilon \rightarrow 0$ in those for $\mu_{\varepsilon}$; namely, in these conditions, one only needs to replace $R(z)$ by

$$
R(z) \cdot \prod_{i=1}^{k}\left(z-a_{i}\right)^{\gamma_{i}} / \prod_{i=1}^{k}\left(z-a_{i}\right)^{\gamma_{i}}
$$

without cancellation to obtain the correct expression. In fact, the algorithm and functional equations obtained in this manner are right in all cases, as the following discussion shows. 
Although the measure $\sigma$ is not balanced, it follows from its definition that it obeys the equation

$$
\begin{aligned}
\int f(z) d \sigma(z) & =\frac{1}{N+M} \sum_{i=1}^{N+M} \int f\left(R_{i}^{-1}(z)\right) d \sigma(z) \\
& =\frac{1}{N+M}\left\{\sum_{i=1}^{N} f\left(R_{i}^{-1}(z)\right) d \sigma(z)+\sum_{j=1}^{k} \gamma_{j} f\left(a_{j}\right)\right\},
\end{aligned}
$$

where for $i=1,2, \ldots, N$, the $\left\{R_{i}^{-1}(z)\right\}$ denote the branches of the inverse of $R(z)$, and the remaining functions are defined by

$$
\begin{aligned}
& R_{N+1}^{-1}(z)=R_{N+2}^{-1}(z)=\cdots=R_{N+\gamma_{1}}^{-1}(z)=a_{1}, \\
& R_{N+\gamma_{1}+1}^{-1}(z)=R_{N+\gamma_{1}+2}^{-1}(z)=\cdots=R_{N+\gamma_{1}+\gamma_{2}}^{-1}(z)=a_{2}, \\
& \cdots \cdots \cdots \cdots \cdots \cdots \\
& R_{N+\gamma_{k-1}+1}^{-1}(z)=R_{N+\gamma_{k-1}+2}^{-1}(z)=\cdots=R_{N+M}^{-1}(z)=a_{k}
\end{aligned}
$$

for all $z$, these representing the "inverses" of

$$
R(z) \cdot \prod_{i=1}^{k}\left(z-a_{i}\right)^{\gamma_{i}} / \prod_{i=1}^{k}\left(z-a_{i}\right)^{\gamma_{i}}
$$

Equation (21) shows, just as it did in the case of $z \rightarrow\left(z^{3}-\lambda z\right) / z$, that as long as $\operatorname{deg} P>\operatorname{deg} Q$ and the moments exist, they can be calculated using (4), (5), and (6) with $z^{N}+a_{1} z^{N-1}+\cdots+a_{N}$ replaced by

$$
P(z) \prod_{i=1}^{k}\left(z-a_{i}\right)^{\gamma_{i}}
$$

$b_{1} z^{N-1}+b_{2} z^{N-2}+\cdots+b_{N}$ replaced by

$$
Q(z) \prod_{i=1}^{k}\left(z-a_{i}\right)^{\gamma_{i}}
$$

and $N$ replaced by $N+M$.

Similarly, we find that the functional equations connected with the condensed measure are always as follows. The spectral function

$$
F(z)=\int_{C} \frac{d \sigma(s)}{z-s}, \quad z \notin C,
$$

obeys

$$
F(z)=\frac{1}{N+M} R^{\prime}(z) F(R(z))+\frac{d}{d z} \ln \left(\prod_{i=1}^{k}\left(z-a_{i}\right)^{\gamma_{i}} Q(z)\right) .
$$

The moment generating function

$$
g(z)=\int_{C} \frac{d \sigma(s)}{1-s z}, \quad \frac{1}{z} \notin C,
$$


obeys

$g(z)=\frac{1}{N+M}\left\{\frac{R^{\prime}(1 / z)}{z R(1 / z)} g\left(\frac{1}{R(1 / z)}\right)+\frac{1}{z}\left[\frac{d}{d w} \ln \left(\prod_{i=1}^{k}\left(w-a_{i}\right)^{\gamma_{i}} Q(w)\right)\right]_{w=1 / z}\right\}$.

When it can be well defined, the potential function

$$
H(z)=\int_{C} \ln (z-s) d \sigma(s)
$$

obeys

$$
H(z)=\frac{1}{N+M}\left\{H(R(z))+\ln \left(Q(z) \prod_{i=1}^{k}\left(z-a_{i}\right)^{\gamma_{i}}\right)\right\} .
$$

We notice the following feature which involves superposition of condensed measures. Suppose $\sigma_{1}$ and $\sigma_{2}$ are associated respectively with

$$
R(z) \prod_{i=1}^{k}\left(z-a_{i}\right)^{\gamma_{i}} / \prod_{i=1}^{k}\left(z-a_{i}\right)^{\gamma_{i}}, \quad R(z) \prod_{i=1}^{\tilde{k}}\left(z-\tilde{a}_{i}\right)^{\tilde{\gamma}_{i}} / \prod_{i=1}^{\tilde{k}}\left(z-\tilde{a}_{i}\right)^{\tilde{\gamma}_{i}},
$$

where $\sum_{i=1}^{k} \gamma_{i}=\sum_{i=1}^{\tilde{k}} \tilde{\gamma}_{i}=M$. Let

$$
F(z)=\int \frac{d \sigma_{1}(s)+d \sigma_{2}(s)}{(z-s)} .
$$

Then

$$
F(z)=\frac{1}{N+M}\left\{R^{\prime}(z) F(R(z))+\frac{d}{d z} \ln \left((Q(z))^{2} \prod_{i=1}^{k}\left(z-a_{i}\right)^{\gamma_{i}} \prod_{i=1}^{\tilde{k}}\left(z-\tilde{a}_{i}\right)^{\tilde{\gamma}_{i}}\right)\right\} \text {. }
$$

Next we turn our attention to a modification of the definition (1) of balanced measures, which applies to a condensed measure $\sigma$. Directly from the definition $(\dagger)$ we have

$$
\sigma\left(R_{i}^{-1}(E)\right)=\sigma(E) /(N+M)
$$

for any branch $R_{i}^{-1}, i=1,2, \ldots, N$, and any Borel subset $E$ such that $a_{j}$ is not an element of $R_{i}^{-1}(E)$ for $j=1,2, \ldots, k$. In [BH] we derived a theorem on the existence and nonexistence of moments of balanced measures based entirely on formula (1) in a special situation. We only need $E$ to lie in an arbitrarily small neighborhood of a fixed point $c$ of $R$, and we consider only the branch of $R^{-1}$ which has $c$ as a fixed point. Equation $(* *)$ for condensed measures applies in such a situation if we take $c=\infty$, which is separated from the points $a_{i}$. All the proof is the same except that $N$ is replaced by $N+M$. Here we restate a special case of Theorem 1 in [BH], but for condensed measures.

THEOREM. Let $\sigma$ be the condensed measure for

$$
R(z) \prod_{i=1}^{k}\left(z-a_{i}\right)^{\gamma_{i}} / \prod_{i=1}^{k}\left(z-a_{i}\right)^{\gamma_{i}}
$$

where $R(z)=\left(z^{N}+a_{1} z^{N-1}+\cdots+a_{N}\right) /\left(b_{1} z^{N-1}+\cdots+b_{N}\right)$. Then the moments

$$
M_{n}=\int_{\hat{\mathbf{C}}} z^{n} d \sigma, \quad n=0,1,2, \ldots,
$$

exist if and only if $\left|b_{1}\right| \leqslant 1$. 


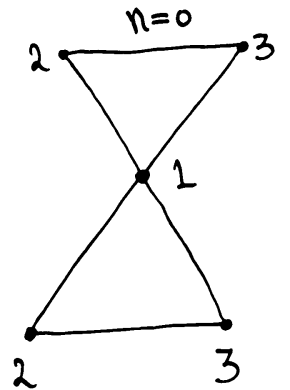

(a)

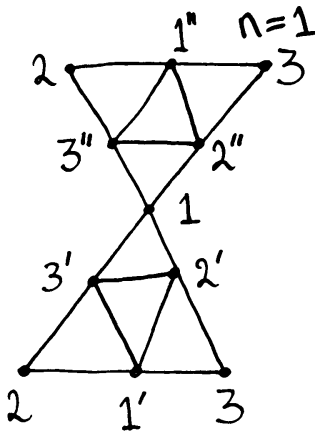

(b)

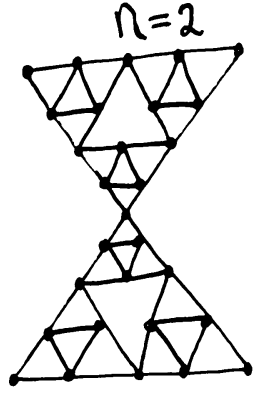

(c)

FIGURE 7. Construction of a Sierpinski lattice. (a) Shows three sites on the largest scale. The boundary conditions identify the corners 2 and 3. (b) By inserting three sites into each of the triangles in (a) the $n=1$ lattice is obtained. (c) Shows the continuation of the procedure at the $n=2$ level. In a similar manner the $n=3,4,5, \ldots$ lattices are constructed.

Let us suppose that $C$ is the condensed Julia set associated with $(\dagger)$ such that the moments all exist, and such that $C \subset \mathbf{R} \cup\{\infty\}$. Then the corresponding orthogonal polynomials, three-term-recurrence formulas and Jacobi matrix $\mathscr{J}$ can all be obtained. Again following [BH] we find that $\mathscr{J}$ is selfadjoint with spectrum $C$ and spectral density $\boldsymbol{\sigma}$.

Condensation leads to no spectral mass associated with the limit points of the point spectra. Other related processes, whereby different branches of the inverse of $R_{\varepsilon}(z)$ are followed with different probabilities, can lead to some spectral mass residing on the derived set of the point spectra.

4. Schrödinger equation on a Sierpinski lattice. Following [DABK] we consider the sequence of fractal lattices shown in Figure 7.

The boundary conditions used identify the corners of the two triangles on the largest scale. The lattice associated with the lower triangle in (a) is generated by inserting three sites that form a small down pointing triangle into each up pointing triangle. Stopping after any finite number $n$ of such insertions, we consider the so-called tight-binding eigenvalue equation in $l_{2}$,

$$
H|\psi\rangle=\varepsilon|\psi\rangle,
$$

where $\varepsilon$ is a dimensionless energy, associated with the Hamiltonian

$$
H=-\sum_{\text {nearest neighbors }}\{|i\rangle\langle j|+| j\rangle\langle i|\} .
$$

Alternatively, one can consider a system of identical masses $m$ placed at the sites of the lattice and connected by springs of strength $k$. The masses move only in a direction orthogonal to the space of the lattice. If one uses $\alpha=m \omega^{2} / k$ to denote a reduced squared frequency and $U_{j} e^{i \omega t}(t=$ time) the displacement of the $j$ th mass at 
time $t$, then the equations of motion are

$$
\alpha U_{i}=\sum_{\text {sites } j \text { connected to } i}\left(U_{i}-U_{j}\right)
$$

which is an eigenvalue problem equivalent to (22) with $\alpha-1=\varepsilon$.

The recursion relation from which the spectrum of $H$ is calculated is obtained by expressing a Schur complement decomposition of the Hamiltonian $H^{(n)}$ at the $n$th step in terms of $H^{(n-1)}$. Following [DABK], the lattice at the $n$th generation is divided into two sublattices, and correspondingly the Hilbert space at the $n$th generation is decomposed into two orthogonal subspaces denotes " 1 " and " 2 ." The set " 1 " is associated with the sites of the $(n-1)$ th lattice, while " 2 " is associated with those sites added to obtain the $n$th lattice from the $(n-1)$ th. Denoting the projection of an eigenfunction $\left|\psi^{(n)}\right\rangle$ of $H^{(n)}$ onto the two subspaces by $\left|\psi_{1}^{(n)}\right\rangle$ and $\left|\psi_{2}^{(n)}\right\rangle$, the eigenvalue equation can be written

$$
\left(\begin{array}{ll}
H_{11}^{(n)} & H_{12}^{(n)} \\
H_{21}^{(n)} & H_{22}^{(n)}
\end{array}\right)\left(\begin{array}{l}
\left|\psi_{1}^{(n)}\right\rangle \\
\left|\psi_{2}^{(n)}\right\rangle
\end{array}\right)=\varepsilon\left(\begin{array}{c}
\left|\psi_{1}^{(n)}\right\rangle \\
\left|\psi_{2}^{(n)}\right\rangle
\end{array}\right) .
$$

Assuming that $\varepsilon$ is not an eigenvalue of $H_{22}^{(n)}$, we obtain from the second equation

$$
\left|\psi_{2}^{(n)}\right\rangle=\left(\varepsilon-H_{22}^{(n)}\right)^{-1} H_{21}^{(n)}\left|\psi_{1}^{(n)}\right\rangle,
$$

and, substituting from this into the first equation,

$$
\left[H_{11}^{(n)}+H_{12}^{(n)}\left(\varepsilon-H_{22}^{(n)}\right)^{-1} H_{21}^{(n)}\right]\left|\psi_{1}^{(n)}\right\rangle=\varepsilon\left|\psi_{1}^{(n)}\right\rangle .
$$

From the last equation, it follows that if $\varepsilon$ is not an eigenvalue of $H_{22}^{(n)}$, namely $\varepsilon \notin\{-2,1\}$, then it is an eigenvalue of $H^{(n)}$ if and only if it is an eigenvalue of the Schur complement operator $H_{11}^{(n)}+H_{12}^{(n)}\left(\varepsilon-H_{22}^{(n)}\right)^{-1} H_{21}^{(n)}$.

What Kadanoff et al. [DABK] show is the remarkable identity

$$
H_{11}^{(n)}+H_{12}^{(n)}\left[\varepsilon-H_{22}^{(n)}\right]^{-1} H_{21}^{(n)}=\frac{4 \varepsilon}{(\varepsilon+2)(\varepsilon-1)} I+\frac{2-\varepsilon}{(\varepsilon+2)(\varepsilon-1)} H^{(n-1)} .
$$

For $\varepsilon$ to be an eigenvalue of this it must be a root of

$$
\begin{gathered}
\operatorname{det}\left[\frac{2-\varepsilon}{(2+\varepsilon)(\varepsilon-1)} H^{(n-1)}+\frac{4 \varepsilon}{(\varepsilon+2)(\varepsilon-1)} I-\varepsilon I\right] \\
=\frac{\varepsilon-2}{(\varepsilon+2)(\varepsilon-1)} \operatorname{Det}\left[H^{(n-1)}+\varepsilon(\varepsilon+3) I\right] .
\end{gathered}
$$

Hence the spectrum of $H^{(n)}$ consists of $\{2\}$ together with the preimages of order one under $z \rightarrow-z \cdot(z+3)$ of the spectrum of $H^{(n-1)}$, with the possible exclusion of preimages in $\{1,-2\}$. Since the spectrum of $H^{(0)}$ is $\{-4,2\}$, since -4 is a fixed point of $z \rightarrow-z \cdot(z+3)$, and since 2 is obtained for all values of $n$, it would appear at first sight that the spectrum of $H^{(n)}$ consists of $\{-4,2\}$ together with all their preimages of all orders up to and including order $n$, and consequently that the spectrum of $\lim _{n \rightarrow \infty} H^{(n)}$, appropriately interpreted, should be the condensed Julia set for $z \rightarrow-z \cdot(z+3)+\varepsilon(z-2)^{-1}+\varepsilon(z+4)^{-1}$. This is not quite the case, 
however, because of the occurrence of the exceptional value 1 among the preimages of -4 and of the exceptional value -2 among the preimages of 2 . The spectrum of $H^{(n)}$ for $n \geqslant 2$ actually consists of

$$
\begin{aligned}
\{-4,2\} & \cup\{\text { preimages of order }(n-1) \text { of }-2\} \\
& \cup\{\text { all preimages of order } \leqslant(n-1) \text { of } \pm 1\} .
\end{aligned}
$$

Hence, the spectrum of $\lim _{n \rightarrow \infty} H^{(n)}$ is actually $C \cup\{-4,2\}$, where $C$ is the condensed Julia set for $z \rightarrow-z \cdot(z+3)+\varepsilon\left(z^{2}-1\right)^{-1}$.

The density of states $\rho^{(n)}(\varepsilon)$ for the Hamiltonian $H^{(n)}$ is obtained by counting the number of states $N^{(n)}(\varepsilon)$ with energy $\varepsilon$ at the $n$th step, and then normalizing using the fact that $H^{(n)}$ is associated with a Hilbert space of dimension $3^{n+1}$, so that $\rho^{(n)}(\varepsilon)=N^{(n)}(\varepsilon) / 3^{n+1}$. Finally, $\rho(\varepsilon)=\operatorname{Lim}_{n \rightarrow \infty} \rho^{(n)}(\varepsilon)$.

In [DABK] it is proved that

(1) $N^{(n)}(-4)=1$ for all $n$,

(2) $N^{(n)}(2)=3^{n}$ for all $n$,

(3) $N^{(n)}(1)=3^{n-1}+1$ for all $n$,

(4) $N^{(n)}(-2)=0$ when $n \neq 1$, and $=1$ when $n=1$,

(5) for all other values of $\varepsilon, N^{(n)}(\varepsilon)=N^{(n-1)}(-\varepsilon \cdot(\varepsilon+3))$.

From these values we readily conclude that

(1) $\rho(-4)=0$,

(2) $\rho(2)=\frac{1}{3}$,

(3) $\rho(1)=\frac{1}{9}$,

(4) $\rho(-2)=0$,

(5) $\rho(\varepsilon)=\frac{1}{3} \rho(-\varepsilon \cdot(\varepsilon+3))$.

Let us state this conclusion slightly differently. $\rho$ associates mass $\frac{1}{9}$ with -1 , mass $\left(\frac{1}{3}\right)\left(\frac{1}{9}\right)$ with each of its two preimages of order 1 under $-z \cdot(z+3), \ldots, \operatorname{mass}\left(\frac{1}{3}\right)^{n} \cdot\left(\frac{1}{9}\right)$ with each of its $2^{n}$ preimages of order $n$ under $-z \cdot(z+3), \ldots$ Let us call this measure $\rho_{1}(\varepsilon)$. $\rho$ also associates mass $\frac{1}{9}$ with +1 , mass $\left(\frac{1}{3}\right)\left(\frac{1}{9}\right)$ with each of its two preimages of order 1 under $-z \cdot(z+3), \ldots, \operatorname{mass}\left(\frac{1}{3}\right)^{n} \cdot\left(\frac{1}{9}\right)$ with each of its $2^{n}$ preimages of order $n$ under $-z \cdot(z+3), \ldots$ Let us call this measure $\rho_{2}(\varepsilon)$. Finally $\rho$ associates mass $\frac{1}{3}$ with 2 ; so that $\rho(\varepsilon)=\rho_{1}(\varepsilon)+\rho_{2}(\varepsilon)+\frac{1}{3} \delta(\varepsilon-2)$. Let $\sigma_{1}=3 \rho_{1}$ and $\sigma_{2}=3 \rho_{2}$. Then $\sigma_{1}$ and $\sigma_{2}$ are the condensed measures for

$$
-z \cdot(z+3)(z+1) /(z+1) \text { and }-z \cdot(z+3)(z-1) /(z-1) \text {, }
$$

respectively. Let $H_{1}(z)$ and $H_{2}(z)$ denote the corresponding potential functions, $H_{i}(z)=\int \ln (z-s) d \sigma_{i}(s)$. Then

$$
\begin{aligned}
& H_{1}(z)=\frac{1}{3} H_{1}(-z \cdot(z+3))+\frac{1}{3} \ln (z+1), \\
& H_{2}(z)=\frac{1}{3} H_{2}(-z \cdot(z+3))+\frac{1}{3} \ln (z-1) ;
\end{aligned}
$$

whence if

$$
\tilde{H}(z)=\frac{1}{3}\left(H_{1}(z)+H_{2}(z)\right)=\int(\ln (z-s))\left(d \rho_{1}(s)+d \rho_{2}(s)\right),
$$

we obtain

$$
\tilde{H}(z)=\frac{1}{3} \tilde{H}(-z \cdot(z+3))+\frac{1}{3} \ln \left(z^{2}-1\right)
$$


Finally, putting

we find

$$
H(z)=\tilde{H}(z)+\frac{1}{3} \ln (z-2)=\int \ln (z-s) d \rho(s)
$$

$$
H(z)=\frac{1}{3} H(-z \cdot(z+3))+\frac{1}{9} \ln \left\{\frac{(z-2)^{3} \cdot(1-z)}{(z+3)}\right\},
$$

which is the functional equation predicted by Rammal [R1, R2], when one notes that his measure has total mass $-\frac{1}{2}$. Rammal uses a different method, derived from statistical mechanics arguments.

We remark that $z \rightarrow-z \cdot(z+3)$ is equivalent to $z \rightarrow z^{2}-\lambda$ with $\lambda=\frac{15}{4}$, upon making a conjugacy transformation $z \rightarrow-z-\frac{3}{2}$.

ACKNOWLEDGEMENTS. P. Moussa is thanked for bringing Sierpinski lattice models to our attention, and for his help in connection with $\S 4$. S. Demko is thanked for many interesting discussions.

\section{REFERENCES}

[AR] R. L. Adler and T. J. Rivlin, Ergodic and mixing properties of Chebychev polynomials, Proc. Amer. Math. Soc. 15 (1964), 794-796.

[Ak] N. I. Akhiezer, The classical moment problem, Hafner, New York, 1965.

[AS] J. Avron and B. Simon, Almost periodic Schrödinger operators, Comm. Math. Phys. 82 (1981), $101-120$.

[BaBM] G. A. Baker, D. Bessis and P. Moussa, A family of almost periodic Schrödinger operators, Los Alamos Preprint, 1981.

[BGH1] M. F. Barnsley, J. S. Geronimo and A. N. Harrington, Orthogonal polynomials associated with invariant measures on Julia sets, Bull. Amer. Math. Soc. (N.S.) 7 (1982), 381-384.

[BGH2] _. On the invariant sets of a family of quadratic maps, Comm. Math. Phys. 88 (1983), 479-501

[BGH3] _ Infinite dimensional Jacobi matrices associated with Julia sets, Proc. Amer. Math. Soc. 88 (1983), 625-630.

[BGH4] Geometry electrostatis measure and orthogonal polynomials on Julia sets for polynomials, J. Ergodic Theory Dynamical Systems 3 (1983), 509-520.

[BGH5] _ Geometrical and electrical properties of some Julia sets, J. Statist. Phys. 37 (1984),

[BGH6]__ Some treelike Julia sets and Pade approximants, Lett. Math. Phys. 7 (1983), 279-286.

[BGH7] _ Almost periodic operators associated with Julia sets, Preprint, Georgia Institute of Technology, 1983; Comm. Math. Phys. (to appear).

[BH] M. F. Barnsley and A. N. Harrington, Moments of balanced measures on Julia sets, Trans. Amer. Math. Soc. 284 (1984), 271-280.

[BT] J. Bellissard and D. Testard, Almost periodic Hamiltonians: an algebraic approach, Preprint, C.N.R.S., Marseille, France, July 1981.

[BBM] J. Bellissard, D. Bessis and P. Moussa, Chaotic states for almost periodic Schrödinger operators, Phys. Rev. Lett. 49 (1982), 701-704.

[Bo] A. N. Berker and S. Ostlund, Renormalization group calculations of finite systems: order parameter and specific heat for epitaxial ordering, J. Phys. C: Solid State Phys. 12 (1979), 4961-4975.

[BGM] D. Bessis, J. S. Geronimo and P. Moussa, Mellin transforms associated with Julia sets and physical applications, J. Statist. Phys. 34 (1984), 15-110.

[BMM1] D. Bessis, M. L. Mehta and P. Moussa, Polynomes orthogonaux sur des ensembles de Cantor et iterations des transformations quadratiques, C. R. Acad. Sci. Paris Sér. I Math. 293 (1981), 705-708.

[BMM2] Orthogonal polynomials on a family of Cantor sets and the problem of iteration of quadratic maps, Lett. Math. Phys. 6 (1982), 123-140.

[BM] D. Bessis and P. Moussa, Orthogonality properties of iterated polynomial mappings, Comm. Math. Phys. 88 (1983). 503-529. 
[BI] P. Blanchard, Complex analytic dynamics on the Riemann sphere, Bull. Amer. Math. Soc. (N.S.) 11 (1984), 85-141.

[Br] H. Brolin, Invariant sets under iteration of rational functions, Ark. Mat. 6 (1965), 103-144.

[C] T. S. Chihara, An introduction to orthogonal polynomials, Gordon \& Breach, New York, 1978.

[DK] R. L. Devaney and M. Krych, Dynamics of $\exp (z)$, Preprint, Boston University, 1983.

[D] S. Demko, On balanced measures (unpublished notes).

[DDI] B. Derrida, L. De Seze and C. Itzykison, Fractal structure of zeros in hierarchical models, J. Statist. Phys. 33 (1983), 559-569.

[DEE] B. Derrida, J. P. Eckmann and A. Erzan, Renormalization groups with periodic and aperiodic orbits, J. Phys. A: Math. Gen. 16 (1983), 893-906.

[DH] B. Derrida and H. Hihlhorst, Singular behaviour of certain infinite products of random matrices, J. Phys. A: Math. Gen. 16 (1983), 2641-2654.

[Dh] D. Dhar, Lattices of effectively non-integral dimensionality, J. Math. Phys. 18 (1977), 577-585.

[DABK] E. Domany, S. Alexander, D. Bensimon and L. P. Kadanoff, Solutions to the Schrödinger equation on some fractal lattices, Phys. Rev. B 28 (1983), 3110-3123.

[Do] A. Douady, Systèmes dynamiques holomorphes, Séminaire Bourbaki, 35e annéé, 1982/83, \#599, 1982.

[DoH] A. Douady and J. Hubbard, Itération des polynômes quadratiques complexés, C. R. Acad. Sci. Paris Sér. I Math. 294 (1982), 123-216.

[F] M. P. Fatou, Sur les equations fonctionelles, Bull. Soc. Math. France 47 (1919), 161-271; ibid. 48 , 33-94; ibid. 48, 208-314.

[FLM] A. Freire, A. Lopes and R. Mañè, An invariant measure for rational maps, Preprint, 1982.

[G] L. Gaal, Classical Galois theory with examples, Markham, Chicago, Ill., 1971.

[Gu] J. Guckenheimer, Endomorphisms of the Riemann sphere, Proc. Sympos. Pure Math. vol. 14, Amer. Math. Soc., Providence, R. I., 1970, pp. 95-123.

[H] M. Herman, Exemples de fractions rationelles ayant une orbite dense sur la sphere de Riemann, Preprint, Ecole Polytechnique, 1983.

[J] G. Julia, Memoire sur l'iteration des fonctions rationelles, J. Math. Pures Appl. 4 (1918), 47-245.

[KG] M. Kaufman and R. B. Griffiths, Exactly soluble Ising models on hierarchical lattices, Phys. Rev. B 24 (1981), 496-498.

[M1] B. Mandlebrot, The fractal geometry of nature, Freeman, San Francisco, Calif., 1982.

[M2] _ Fractal aspects of the iteration of $z \rightarrow \lambda z(1-z)$, Ann. N.Y. Acad. Sci. 357 (1980), 249-259.

[Ma] R. Mañè, On the uniqueness of the maximizing measure for rational maps, Preprint, Rio de Janeiro, 1982.

[MSS] R. Mañè, P. Sad and D. Sullivan, On the dynamics of rational maps, Preprint.

[NF] D. R. Nelson and M. E. Fisher, Soluble renormalization group and scaling fields for low dimensional Ising systems, Ann. Physics 91 (1975), 226-274.

[PK] T. S. Pitcher and J. R. Kinney, Some connections between ergodic theory and iteration of polynomials, Ark. Mat. 8 (1968), 25-32.

[R1] R. Rammal, On the nature of eigenstates on fractal structures, Phys. Rev. B 28 (1983), 4871-4874.

[R2] , Spectrum of harmonic excitations on fractals, J. Physique (Paris) 45 (1984), 191-206.

[Re] M. Rees, Ergodic rational maps with dense critical point-forward orbit, University of Minnesota Mathematics Report, 82-140.

[Ru] D. Ruelle, Repellers for real analytic maps, Ergodic Theory Dynamical Systems 2 (1982), 99-108.

[S1] D. Sullivan, Iteration des fonctions analytiques complexes, C. R. Acad. Sci. Paris Sér. I Math. 294 (1982), 301.

[S2] _ Solution of the Fatou-Julia problem on wandering domains, Preprint, 1982.

[S3] _ _ Structural stability implies hyperbolicity for Kleinian groups, Preprint, 1982.

[S4] _. Topological conjugacy classes of analytic endomorphisms, Preprint, 1982.

[Sz] G. Szego, Orthogonal polynomials, Amer. Math. Soc., Providence, R.I., 1939.

[T] W. Thurston, Lecture Notes, CBMS Conf., Univ. of Minnesota, 1983.

School of Mathematics, Georgia Institute of Technology, Atlanta, Georgia 30332 (Current address of M. F. Barnsley and J. S. Geronimo)

Department of Mathematical Sciences, Loyola University, Chicago, Illinois 60627 (Current address of A. N. Harrington) 